\title{
TGF- $\beta 1$ stimulates epithelial-mesenchymal transition and cancer-associated myoepithelial cell during the progression from in situ to invasive breast cancer
}

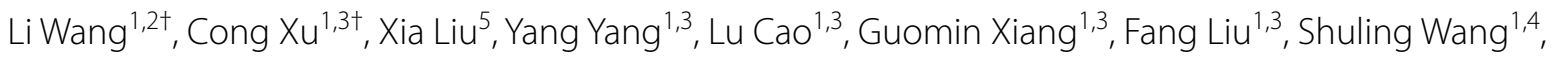
Jing Liu, ${ }^{1,3}$, Qingxiang Meng ${ }^{1,3}$, Jiao Jiao ${ }^{1,3}$ and Yun Niu, ${ }^{1,3^{*}}$ (D)

\begin{abstract}
Background: The progression of ductal carcinoma in situ (DCIS) into invasive ductal carcinoma (IDC) is prevented by normal breast myoepithelial cells. Studies have suggested that EMT-associated genes were enriched in IDC in contrast to DCIS. This paper explored the relationship and potential mechanism between myoepithelial cells and EMT-associated genes in facilitating the transformation from DCIS to breast cancer.

Methods: EMT markers and myoepithelial phenotypic markers in IDC, DCIS, and healthy breast tissue were characterized using immunohistochemical assay. Both in vivo and in vitro models were created to mimic the various cell-cell interactions in the development of invasive breast cancer.

Results: We found that EMT markers were more abundant in invasive carcinomas than DCIS and adjacent normal breast tissue. Meanwhile, TGF- $\beta 1$ regulated the morphology of MCF-7 (epithelial cells substitute) migration and EMT markers during the transformation from DCIS to invasive breast cancer. Additionally, TGF- $\beta 1$ also regulated invasion, migration and cytokines secretion of MDA-MB-231 (myoepithelial cells substitute) and epithelial cells when co-cultured with MCF-7 both in vitro and in vivo.
\end{abstract}

Conclusions: In conclusion, these findings demonstrated that both EMT phenotypes and cancer-associated myoepithelial cells may have an impact on the development of invasive breast cancer.

Keywords: DCIS, Progression, Epithelial-mesenchymal transition, Myoepithelial cell, TGF- $\beta 1$

*Correspondence: yunniu0823@126.com

†Li wang and Cong Xu contributed equally to this work

${ }^{1}$ Tianjin Medical University Cancer Institute and Hospital, National Clinical

Research Center of Cancer, Key Laboratory of Breast Cancer Prevention and Therapy, Tianjin Medical University, Ministry of Education, Key

Laboratory of Cancer Prevention and Therapy, West Huanhu Road, Ti Yuan Bei, Hexi District, Tianjin 300060, China

Full list of author information is available at the end of the article

\begin{abstract}
Introduction
Ductal carcinoma in situ (DCIS) is recognized as a localized tumor cell proliferation in the ductal-lobular system that does not penetrate the basement membrane and has the potential to transform into invasive breast cancer [1]. The cascade of events that occur between benign and malignant transformation has not been sufficiently clarified and is a complex process dependent of both the microenvironment as well as the tumor cell properties [2, 3].
\end{abstract}

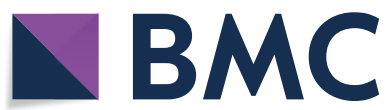

(c) The Author(s) 2019. This article is licensed under a Creative Commons Attribution 4.0 International License, which permits use, sharing, adaptation, distribution and reproduction in any medium or format, as long as you give appropriate credit to the original author(s) and the source, provide a link to the Creative Commons licence, and indicate if changes were made. The images or other third party material in this article are included in the article's Creative Commons licence, unless indicated otherwise in a credit line to the material. If material is not included in the article's Creative Commons licence and your intended use is not permitted by statutory regulation or exceeds the permitted use, you will need to obtain permission directly from the copyright holder. To view a copy of this licence, visit http://creativeco mmons.org/licenses/by/4.0/. The Creative Commons Public Domain Dedication waiver (http://creativecommons.org/publicdomain/ zero/1.0/) applies to the data made available in this article, unless otherwise stated in a credit line to the data. 
One such process that is known to be involved in carcinogenesis is the epithelial-mesenchymal transition (EMT). EMT occurs when epithelial cells acquire mesenchymal properties such as cytoskeleton reorganization, loss of cell polarity and breakdown of cell junctions-all of which lead to increased cell motility $[4,5]$. Besides carcinogenesis, this process has also been demonstrated in tissue regeneration and wound healing [6]. Both local and disseminated tumor metastasis have been thought to be a product of the EMT, as this process bestows otherwise benign cells with the properties to escape the rigid constraints of the surrounding tissue architecture, such as the basement membrane. This process was instigated as a result of several extracellular stimuli of which transforming growth factor- $\beta$ (TGF- $\beta$ ) played a predominant role [7-9]. Recent literature has documented an increase in EMT-related gene expression in invasive cancer in comparison to DCIS $[10,11]$. Nevertheless, data on the expression of EMT markers in DCIS and invasive carcinoma is scarce.

Normal mammary gland physiology and development are highly dependent on myoepithelial cells which surround mammary ducts and lobular acini $[12,13]$. These cells possess properties that naturally act to suppress tumor formation such as the ability to maintain epithelial cell polarity, providing a physical barrier between epithelial cells and the surrounding stroma and ensuring the integrity of the ductal-lobular basement membrane [14]. Nevertheless, the functional and phenotypical differences between normal breast tissue myoepithelial cells and DCIS-associated myoepithelial cells in the context of malignant transformation are not known. A majority of literature on the topic have instead focused more on luminal epithelial cells, although a number of molecular studies have suggested that there are differences between normal breast tissue myoepithelial cells and DCIS-associated myoepithelial cells that may be underlie latter's propensity for malignant transformation [15, 16].

The current investigation explores the expression of EMT markers (N-cadherin, Snail, Twist, Vimentin, Zeb1, E-cadherin) in invasive carcinomas and DCIS. The functional and immunophenotypic characteristics of DCISassociated myoepithelial cells were also assessed through myoepithelial cell phenotypic markers (Calponin, SMA, p63). Subsequent investigation showed that stimulation with TGF- $\beta 1$ induced EMT in MCF-7. Cell-based assays were carried out to document the cascade of cell-cell interaction during the evolution from non-malignant to malignant. We originally used this co-culture system and other methods to demonstrate the TGF- $\beta 1$ role between epithelial and myoepithelial cells in development of preinvasive breast cancer both in vitro and in vivo. All the resulting experimental data indicated that TGF- $\beta 1$ has a significant role in the transformation from premalignant to invasive breast cancer.

\section{Materials and methods \\ Patient samples and clinical profiles}

116 and 88 cases of formalin-fixed and paraffin-embedded surgical samples of breast IDC and DCIS respectively chose between 1 January 2004 and 31 December 2006 from patients treated in the Tianjin Medical University Cancer Institute and Hospital. This series is significant as it comprises a large cohort of patients under long-term monitoring in a single institution. All patients were women between the ages of 25 and 82 years (average of 48 years). Table 1 depicts other clinical characteristics. None of these patients had undergone neoadjuvant chemotherapy. Three pathologists (Yun Niu., Xiaolong Feng, and Shuhua Lv.) were involved in reviewing the histopathological results and diagnoses in accordance to the World Health Organization criteria. All patients provided written informed consent prior to any surgical procedures and sample collection. The study protocol was reviewed by the Human Ethical Committee of Tianjin Medical University Cancer Institute and Hospital.

\section{Immunohistochemistry}

$4 \mu \mathrm{m}$ thick slices of paraffin-embedded tissues were first sectioned. Paraffin was then removed from these sections before they were rehydrated and exposed overnight to primary antibodies at $4{ }^{\circ} \mathrm{C}$. A broad-spectrum secondary antibody was then added onto these slices before treatment with DAB. Visualization of the final product with a light microscope was done after hematoxylin counterstaining. Immunostaining-based scores were done by three pathologists who were blinded to the clinical background of each patients' sample. The location, intensity and percentage of immunoreactivity, were evaluated for each antibody. Staining intensities were graded from a scale of zero to three: ( 0 , no reactivity; 1 , weak reactivity; 2 , moderate reactivity; 3 , strong reactivity) while positive cell percentage were grouped into four cohorts: 0 (no tumor cells), 1 (1-25\%), 2 (26-50\%), 3 (51-75\%), 4 $(>76 \%)$. The sum of grades of staining intensity and percentage of positive cells were (0-2, negative vs. $3-7$, positive) for E-cadherin, N-cadherin, Snail, Twist, Vimentin, Zeb1, SMA, p63, and Calponin. Ki67 status was illustrated in terms of percentage of positive cells, with a threshold of $20 \%$ of positive cells, ER and PR were interpreted as positive if more than $1 \%$ of tumor cells demonstrated positive nuclear staining and whole membrane strong staining of HER $2+$ was determined when more than $10 \%$ of the tumor cells were strongly stained. 
Table 1 Clinical information for invasive ductal carcinoma (IDC) and ductal carcinoma in situ (DCIS)

\begin{tabular}{|c|c|c|c|c|}
\hline \multirow[t]{2}{*}{ Characteristic } & \multicolumn{2}{|c|}{$\operatorname{DCIS}(n=88)$} & \multicolumn{2}{|c|}{$\operatorname{IDC}(n=116)$} \\
\hline & No. & $\%$ & No. & $\%$ \\
\hline \multicolumn{5}{|c|}{ Age at diagnosis (years) } \\
\hline$\leq 50$ & 46 & 52.3 & 80 & 69 \\
\hline$>50$ & 42 & 47.7 & 36 & 31 \\
\hline \multicolumn{5}{|l|}{ Tumor size (cm) } \\
\hline$\leq 2$ & 27 & 30.7 & 35 & 30.2 \\
\hline $2-5$ & 58 & 65.9 & 69 & 62.3 \\
\hline$>5$ & 3 & 3.4 & 12 & 7.4 \\
\hline \multicolumn{5}{|c|}{ Histological grade } \\
\hline 1 & & & 20 & 17.2 \\
\hline 2 & & & 77 & 66.4 \\
\hline 3 & & & 19 & 16.4 \\
\hline \multicolumn{5}{|l|}{ Nuclear grade } \\
\hline 1 & 24 & 27.3 & & \\
\hline 2 & 50 & 56.8 & & \\
\hline 3 & 14 & 15.9 & & \\
\hline \multicolumn{5}{|c|}{ Lymph node status } \\
\hline 0 & 88 & 100 & 55 & 47.4 \\
\hline $1-3$ & 0 & 0 & 37 & 31.9 \\
\hline $4-9$ & 0 & 0 & 12 & 10.3 \\
\hline$\geq 10$ & 0 & 0 & 12 & 10.3 \\
\hline \multicolumn{5}{|l|}{ ER status } \\
\hline Negative & 20 & 22.7 & 50 & 43.1 \\
\hline Positive & 68 & 77.3 & 66 & 56.9 \\
\hline \multicolumn{5}{|l|}{ PR status } \\
\hline Negative & 23 & 26.1 & 62 & 53.4 \\
\hline Positive & 65 & 73.9 & 54 & 46.6 \\
\hline \multicolumn{5}{|l|}{ Her-2 } \\
\hline Negative & 56 & 63.6 & 70 & 60.3 \\
\hline Positive & 32 & 36.4 & 46 & 39.7 \\
\hline \multicolumn{5}{|l|}{ ki67 } \\
\hline$<20$ & 65 & 73.9 & 20 & 17.2 \\
\hline$\geq 20$ & 23 & 26.1 & 96 & 82.8 \\
\hline
\end{tabular}

\section{Cell co-culture conditions and treatments}

The Cell Bank of the Chinese Academy of Sciences (Shanghai, China) provided both cell lines MDA-MB-231 and MCF-7 which were cultured in RPMI-1640 mixed with $100 \mu \mathrm{g} / \mathrm{ml}$ each of streptomycin and penicillin as well as $10 \%$ fetal bovine serum. For investigating the paracrine effects of the cells, we used the Transwell insert system (Fig. 1). This system comprises of two compartments that were divided by a semi-permeable fenestrated membrane with $0.4 \mu \mathrm{m}$ pore sizes (BD Biosciences, USA). The lower compartment contained MDA-MB-231 cells $\left(3 \times 10^{5} \mathrm{cell} /\right.$ per well, in 6 -well plates $)$ while the upper chamber contained MCF-7 cells $\left(3 \times 10^{5}\right.$ cell/per well,

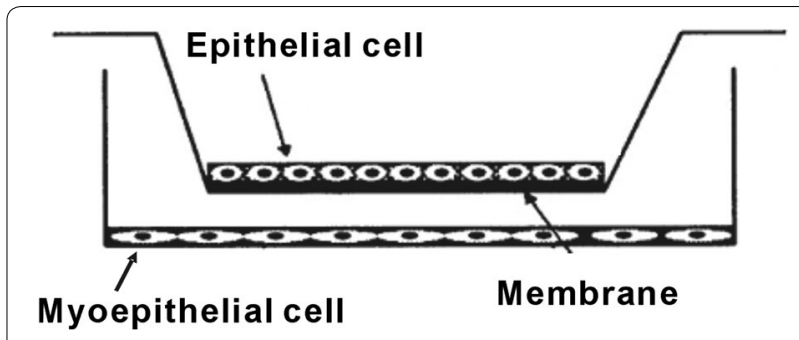

Fig. 1 Experimental model. Epithelial cell and myoepithelial cell were co-cultured in the Transwell insert system

in 6-well plates). These cells were co-cultured for 3 days under a humidified condition with $5 \% \mathrm{CO}_{2}$ and at a temperature of $37^{\circ} \mathrm{C}$.

Our experiments consisted of three separate cell cohorts: MDA-MB-231 cells alone (MDAMB-231+Ctrl), MCF-7+ MDA-MB-231 cells (Co-culture + Ctrl), and MCF-7 + MDA-MB-231 cells + TGF- $\beta 1$ $10 \mathrm{ng} / \mathrm{ml}$ (Co-culture + TGF- $\beta \mathrm{l})$. MCF-7 cells were subjected to a 24-hour exposure to TGF- $\beta 1$ at $10 \mathrm{ng} / \mathrm{ml}$ prior to co-culture with MDA-MB-231 cells. TGF- $\beta 1$ (100-21) was purchased from Pepro Tech (USA).

\section{Western blot}

Cells were rinsed thrice with PBS before undergoing RIPA buffer and $1 \mathrm{mM}$ PMSF- facilitated cell lysis. The resultant substrate was centrifuged and Western blot analysis was carried out on the supernatant fractions. These cell proteins were mixed into a SDS-PAGE membrane with $10 \%$ gel and subjected to electrophoresis. Protein bands in the gel were then immunoblotted onto polyvinylidene difluoride (PVDF) membranes before being probed with the aforementioned primary antibodies followed by secondary antibodies. ECL regents were used to detect positive bands of the blot.

\section{Immunofluorescence assay}

Immunofluorescence assay was carried out as previously described [17]. CD10 (Bioss, China) CK8/18 (Thermo Fisher) were utilized for study. Immunofluorescence assays were visualized using Goat anti-Rabbit Alexa Fluor ${ }^{\circledR}$ 594-conjugated (Zhongshan gold bridge) or Goat anti-Mouse Fluorescein-conjugated (Zhongshan gold bridge) antibodies. Cell nuclei were counterstained using DAPI (Thermo Fisher Scientific). Representative images were captured and analyzed by microscopy.

\section{RNA extract and qRT-PCR}

Trizol reagent (Takara, Japan) was used to isolate total RNA from cell cultures in compliance to manufacturer's protocols. Optical density $\left(\mathrm{A}_{260}\right)$ methods were used to 
quantify RNA prior to its storage at $-80{ }^{\circ} \mathrm{C}$ until further usage. The Reverse Transcription System (TAKARA, Dalian, China) using oligo (dT) primers allowed for reverse transcription of RNA into cDNA. $\beta$-actin was used as a housekeeping gene to verify cDNA integrity. The single-stranded cDNA was amplified using the following primers:E-cadherin: Forward:5'-TCCATTTCT TGGTCTACGCC-3', Reverse:5'-CACCTTCAGCCA TCCTGTTT-3'; N-cadherin: Forward:5'-ACAGTGGCC ACCTACAAAGG-3', Reverse: 5'-CCGAGATGGGGT TGATAATG-3'; Vimentin: Forward: 5'-CCTTGAACG CAAAGTGGAATC-3'; Reverse: 5'-GACATGCTGTTC CTGAATCTGAG- $3^{\prime}$; $\beta$-actin Forward: $5^{\prime}$-TCGTGCGTG ACATTAAGGAG-3'; Reverse: 5'-ATGCCAGGGTAC ATGGTGGT- $3^{\prime}$. $\beta$-actin was used as an internal control. The relative gene expression was calculated by the $2^{-\Delta \Delta \mathrm{Ct}}$.

\section{MTT assays}

Cell proliferation assay was carried out with the 3-(4,5-dimethylthiazol-2-yl)-2,5-diphenyltetrazolium bromide (MTT) assay. Each well of a 96-well plate were seeded with $5 \times 10^{3}$ cells. The cells first underwent primary incubation before being incubated for $4 \mathrm{~h}$ with $20 \mu \mathrm{l} \mathrm{MTT}$ at $37^{\circ} \mathrm{C}$. The medium was then rinsed out and $150 \mu \mathrm{l}$ DMSO was used to dissolve precipitated Formosan. A micro-plate auto-reader (Bio-Rad, USA) was used to detect absorbance at $490 \mathrm{~nm}$.

\section{Wound healing assay}

$3 \times 10^{5}$ cells were seeded in a $35 \mathrm{~mm}$ culture dish. A single scratch was inflicted with a $200 \mu \mathrm{l}$ pipette tip on the cell monolayer (formed after allowing cells to proliferate for $24 \mathrm{~h}$ ). The cells were imaged under phase-contrast microscopy at time zero and immediately post incision.

\section{Preparation of the conditioned medium}

Media that was pre-conditioned in the Transwell insert systems were aspirated after $72 \mathrm{~h}$, filtered through $0.2 \mu \mathrm{m}$ pores and stored at $-80^{\circ} \mathrm{C}$.

\section{Transwell invasion assays}

Transwell invasion assay was carried out in modified Matrigel-coated 24-well chambers. The lower chamber contained $3 \times 10^{5}$ MCF-7 cells while the upper chamber contained $3 \times 10^{5}$ MDA-MB-231 cells in serum-free medium. Cells were allowed to migrate during a 48-hour incubation period. Cells in the lower chamber with $10 \%$ were then counted across five randomly selected fields.

\section{ELISA}

ELISA kit (R\&D Systems) experiments were carried out using the conditioned media and were done in compliance to manufacturer's instructions. The conditioned supernatants were collected from the Transwell cocultures after $72 \mathrm{~h}$ of co-culture in order to determine the levels of levels of MCF-7 and MDA-MB-231 secreted cytokines, which included MMP-9 and IL-6. A microplate reader was used to measure the absorbance at $450 \mathrm{~nm}$ of each well. Standard curves were used to calculate the concentrations of chemokine protein concentrations.

\section{Xenograft mouse model}

30, six weeks old female BALB/c nude mice weighing between 16 and $18 \mathrm{~g}$ each were purchased and feet in specific pathogen free (SPF) conditions. Procedures of animal care were passed by the institutional animal use and care committee of Tianjin Medical University Cancer Institute and hospital. Three independent groups of cells were treated as above. MDA-MB-231 cells alone (MDA-MB-231+Ctrl), MCF-7+MDA-MB-231 cells (Co-culture $+\mathrm{Ctrl}$ ), and MCF-7 + MDA-MB-231 cells + TGF- $\beta 110 \mathrm{ng} / \mathrm{ml}$ (Co-culture + TGF- $\beta \mathrm{l}) .2 \times 10^{6}$ MDA-MB-231 cells were administered into the fat pads of nude mice. Tumor volumes were measured at an interval of every 5 days post-tumor cells injection. All animals were sacrificed after 40 days and all tumors were collected and weighted. All tumors and livers, lungs were fixed in formalin for further hematoxylin-eosin staining (HE). Tumor volume was calculated using the formula: (length $\times$ width $\left.^{2}\right) / 2$.

\section{Statistical analysis}

The SPSS software (ver. 16; Chicago, IL, USA) was used to perform all statistical analyses. Immunohistochemical data were analyzed using $X^{2}$ test. Data are presented as mean \pm standard deviation of three duplicated tests. Differences between groups were compared using Student's $\mathrm{t}$-test and one-way ANOVAS. Statistical significance was considered at $p<0.05$.

\section{Results \\ Expression of EMT markers in DCIS, IDC, and adjacent normal breast tissue}

The loss of E-cadherin and increased expression of mesenchymal markers (N-cadherin, Snail, Twist, Vimentin, and Zeb1) were used as EMT indicators in breast cancer cells (Fig. 2). Invasive lobular carcinomas were not included in this study as they are characterized by loss of E-cadherin. N-cadherin, Snail, Twist, Vimentin, and Zeb1 were expressed in 19.8\% (23/116), 19\% (22/116), $9.5 \%(11 / 116), 16.4 \%(19 / 116)$, and $12.9 \%(15 / 116)$ of the invasive carcinomas. There was a loss of E-cadherin in 27.6\% (32/116) of the invasive carcinoma samples (Fig. 3). In DCIS, N-cadherin, Snail, Twist, Vimentin, and Zeb1 were expressed in $5.7 \%(5 / 88), 3.4 \%$ (3/88), $2.3 \%(2 / 88)$, 


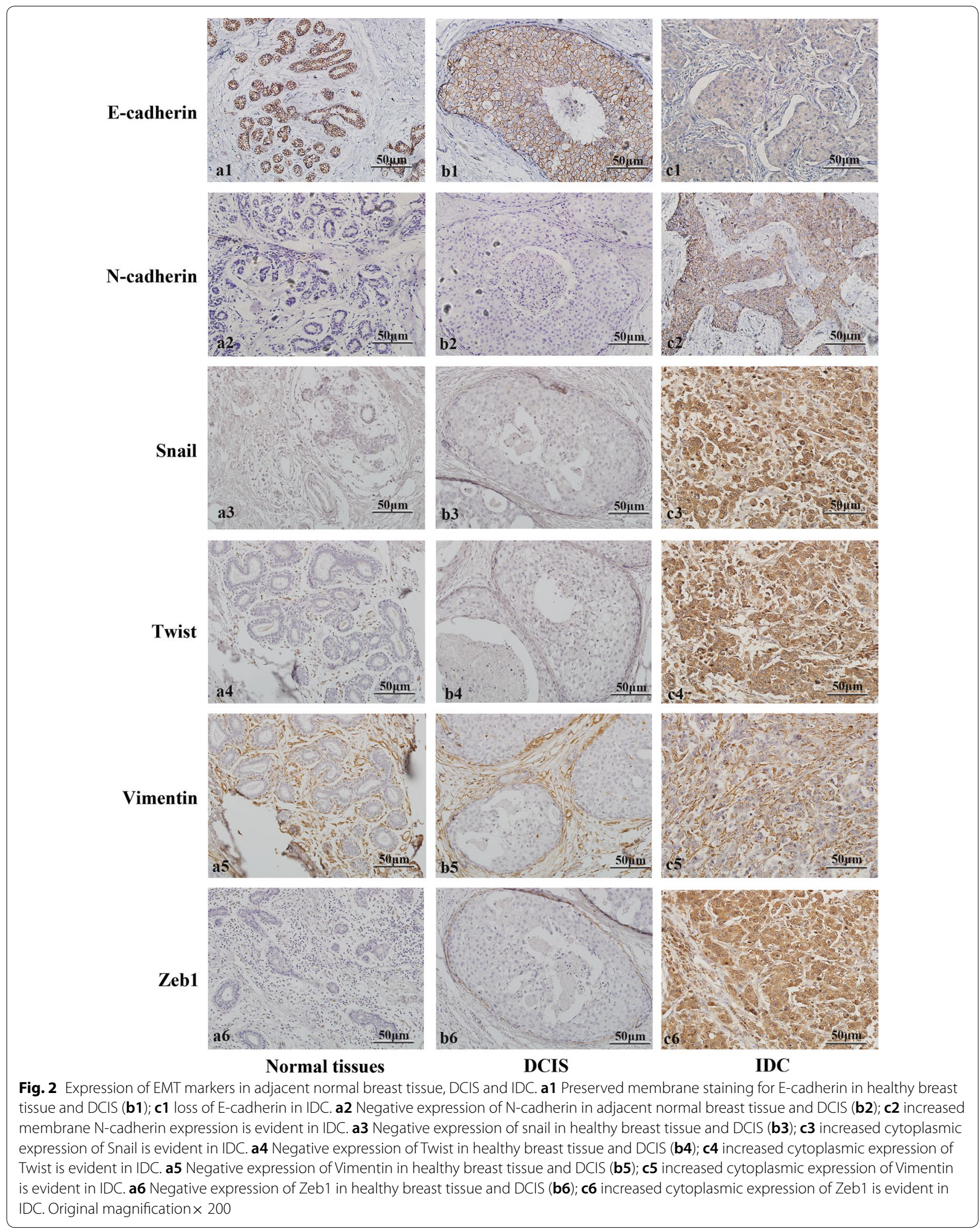



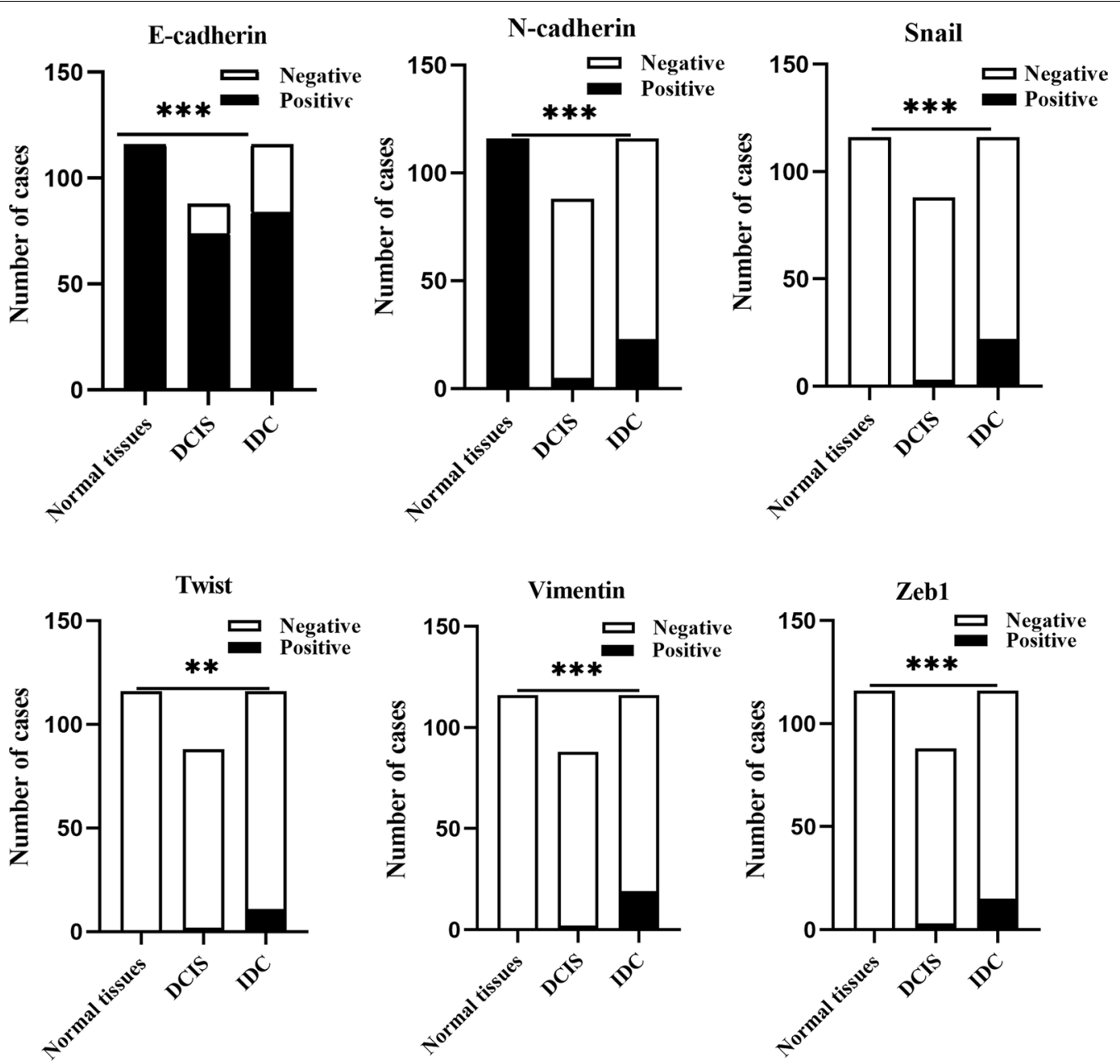

Fig. 3 Expression of EMT markers in adjacent normal breast tissue, DCIS and IDC. ${ }^{* *} p<0.01,{ }^{* * *} p<0.001$

$2.3 \%(2 / 88)$, and $3.4 \%(3 / 88)$. E-cadherin loss was found in $15.9 \%(14 / 88)$ (Fig. 3). None of the adjacent normal breast tissue expressed N-cadherin, Snail, Twist, Vimentin, or Zeb1 and none of them displayed a loss of E-cadherin (Fig. 3).

The expressions of N-cadherin, Snail, Twist, Vimentin and Zeb1, and number of cells that demonstrated loss of E-cadherin were significantly higher in invasive carcinomas in contrast to normal tissue and DCIS samples $(p<0.05$; Fig. 3).

\section{Phenotypic myoepithelial cell markers in adjacent normal breast tissue, DCIS and IDC}

All one hundred and sixteen healthy breast tissue specimens $(100 \%)$ and all eighty-eight DCIS (100\%) specimens demonstrated strong SMA expressions in the myoepithelial cells (Fig. 4). However, none of the IDC samples expressed SMA (Fig. 4). All myoepithelial cells of 116 healthy normal breast tissue specimens demonstrated strong and continuous P63 expression (100\%) (Fig. 4). In DCIS cells, p63 expression was continuous in $77 / 88$ (87.5\%) specimens, discontinuous in $5(5.7 \%)$ specimens, and absent in $6(6.8 \%)$ specimens. None of the IDC expressed p63 (Fig. 4d). Similarly, all healthy breast tissue samples demonstrated strong calponin expression (100\%) (Fig. 4). 83 (94.3\%) of DCIS specimens had significant Calponin expression, two (2.3\%) samples had discontinuous calponin expressions and $3(3.4 \%)$ specimens did not express calponin. None of the IDC expressed Calponin (Fig. 4d).

The differences in SMA, p63, and Calponin expressions in myoepithelial cells among healthy breast tissue, DCIS and IDC were statistically significant $(p<0.05$, respectively; Fig. 4d).

\section{Identification of cell phenotype}

Breast cancer cell lines representing epithelial cells (MCF7) and myoepithelial cells (MDA-MB-231) were 


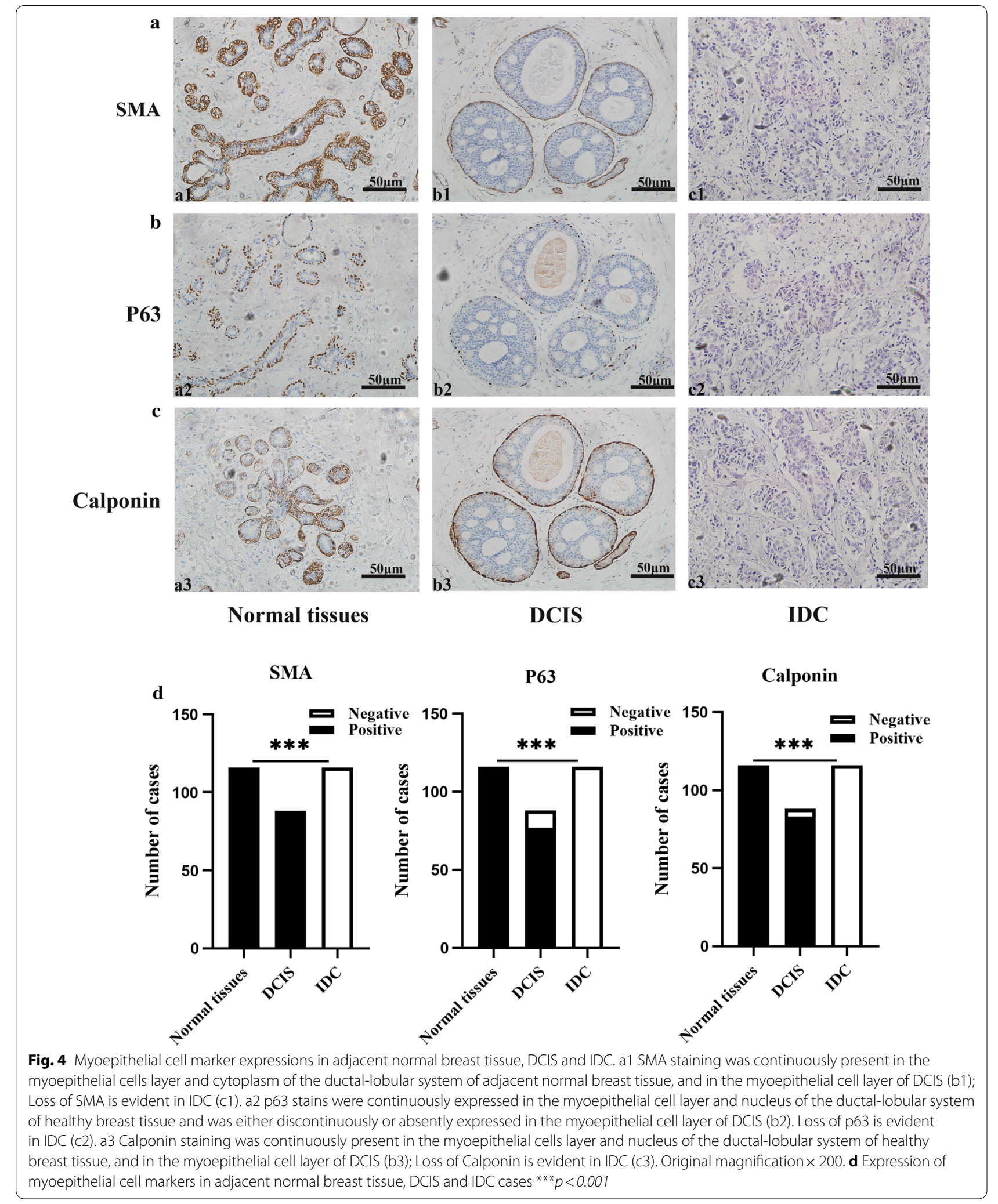


used in this study. Firstly, immunofluorescence assay and western blotting were used to quantify cell phenotype. MCF7 cells demonstrated no CD10 and CK5/6 but high levels of $\mathrm{CK} 8 / 18$, while the converse results were seen in MDA-MB-231 cells (Fig. 5a, b). These findings confirm the viability of MDA-MB-231 as a representative of myoepithelial cells and MCF7 as a representative of epithelial cells.

\section{TGF- $\beta 1$ regulates the morphology, migration and EMT} markers of MCF-7

MCF-7 cells demonstrated cell-cell adhesion and epithelial-like morphology. Upon exposure to TGF- $\beta 1$, there was a change of cell morphology from oval to spindle shape as well as a decrease in cell-cell adhesion (Fig. 5c). Additionally, there was a reduction of E-cadherin at the protein and mRNA levels, and an increase in mesenchymal markers N-cadherin and Vimentin in MCF-7 expressions (Fig. 5d, e). The induced motility and migration of TGF- $\beta 1$ were not a result of reduced cell viability as confirmed the cell proliferation (MTT assay) which

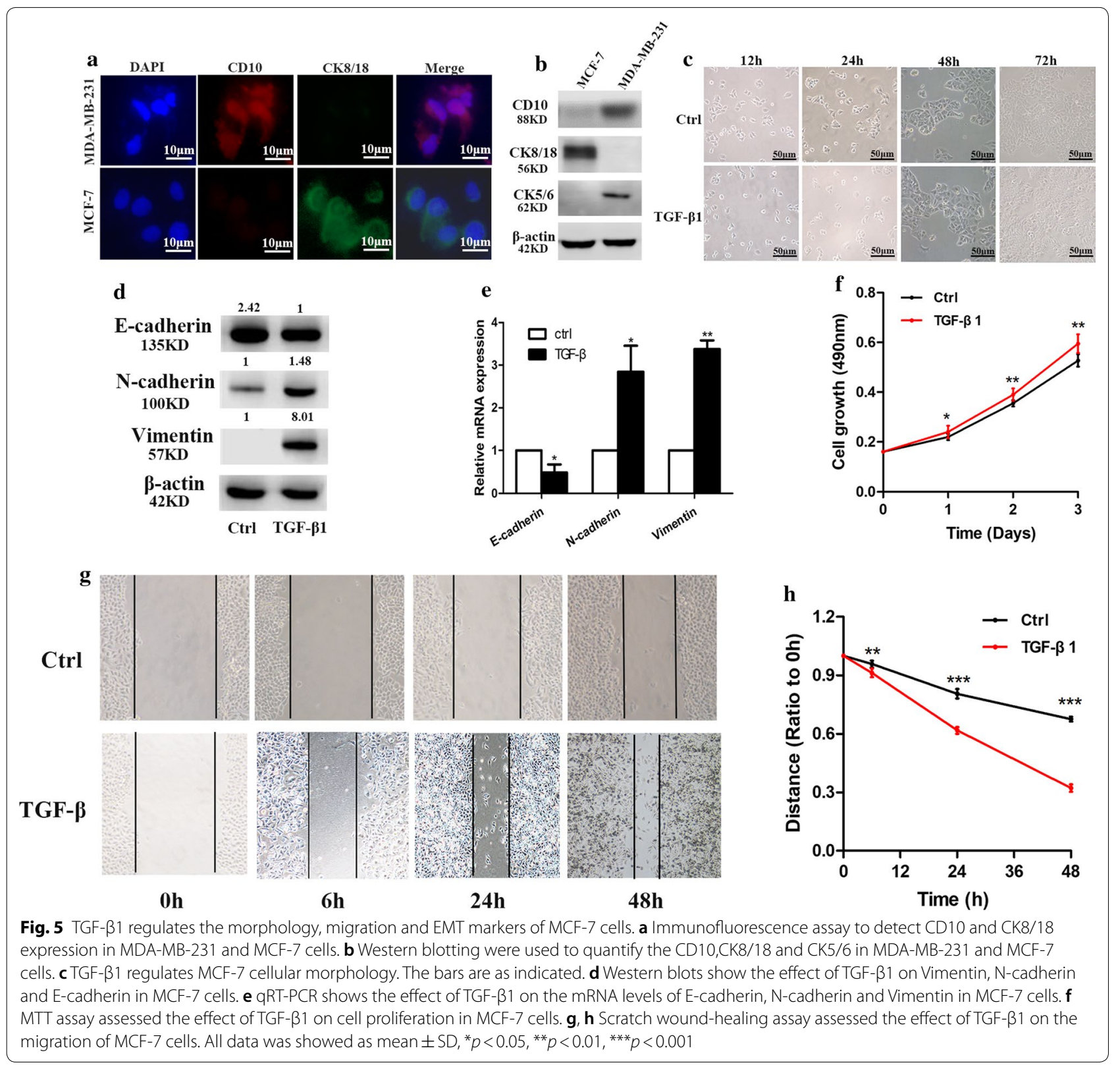


(See figure on next page.)

Fig. 6 TGF- $\beta 1$ regulates migration, invasion and cytokines secretion of MDA-MB-231 cells when co-cultured with MCF-7 cells. a MDA-MB-231 cellular morphology remained. The bars are as indicated. b MTT assay evaluated TGF- $\beta 1$ effects on MDA-MB-231 cell proliferation when co-cultured with MCF-7 cells. $\mathbf{c}$ Scratch wound-healing assay assessed TGF- $\beta 1$ effects of MDA-MB-231 cell migration when co-cultured with MCF-7 cells. d

Transwell assay assessed TGF- $\beta 1$ effects on the invasion of MDA-MB-231 cells when co-cultured with MCF-7 cells. e MMP-9 and IL-6 results obtained by the ELISA. All data was showed as mean $\pm S D,{ }^{*} p<0.05,{ }^{* *} p<0.01,{ }^{* * *} p<0.001$

demonstrated no change despite exposure to TGF- $\beta 1$ (Fig. 5f). A wound-healing assay was used to determine the influence of TGF- $\beta 1$ on cell migration. Stimulation with TGF- $\beta 1$ in MCF-7 significantly enhanced the migration of cells in contrast to control cells $(p<0.05)$ (Fig. $5 \mathrm{~g}$, h). Taken together, TGF- $\beta 1$ is a critical factor that regulates MCF-7 morphology, migration and expression of EMT markers during the progression from in situ to invasive breast cancer.

TGF- $\beta 1$ regulates migration, invasion and cytokines secretion of MDA-MB-231 when co-cultured with MCF-7 MDA-MB-231 remained spindle shape when co-cultured with MCF-7 stimulated with TGF- $\beta 1$ (Fig. 6a), while the MTT assay demonstrated no change despite stimulation with TGF- $\beta 1$ (Fig. 6b). Co-culture + TGF- $\beta 1$ group significantly enhanced the migration of cells compared with MDA-MB-231 alone and Co-culture + Ctrl group $(p<0.05)$ (Fig. 6c). In addition, we found similar results in Transwell assay $(p<0.05)$ (Fig. 6d). The Co-culture + TGF- $\beta 1$ group produced higher levels of MMP-9 and IL- 6 than the MDA-MB-231 alone and the Co-culture $+\mathrm{Ctrl}$ group $(p<0.05)$ (Fig. 6e). Co-culture $+\mathrm{Ctrl}$ and MDA-MB-231 + Ctrl produced an equal amount of MMP-9 and IL- 6 when compared to levels produced by MCF-7 alone. Co-culture + Ctrl demonstrated the impact on MCF-7 on MDA-MB-231 in the co-culture system. We concluded that TGF- $\beta 1$ regulates migration, invasion, and cytokines secretion of MDA-MB-231 throughout the evolution from in situ to invasive breast cancer when cocultured with MCF-7.

TGF- $\beta 1$ effectively promoted tumor growth and metastasis when MDA-MB-231 co-cultured with MCF-7 compared with the other two groups

To verify the function of TGF- $\beta 1$ in vivo, three groups are conducted: MDA-MB-231 cells alone (MDAMB-231 + Ctrl), MCF-7+MDA-MB-231 cells (Co-culture + Ctrl), and MCF-7 + MDA-MB-231 cells + TGF- $\beta 1$ $10 \mathrm{ng} / \mathrm{ml}$ (Co-culture + TGF- $\beta \mathrm{l})$ (Fig. 7a-c). Nude mice of Co-culture + TGF- $\beta$ l were found to have much larger tumors than MDA-MB-231 + Ctrl and Co-culture + Ctrl (Left panels of Fig. 7a-c). There were significant differences in tumor volumes between MDA-MB-231+Ctrl and Co-culture + Ctrl or Co-culture + TGF- $\beta 1$ (Fig. 7f) as validated by the weights of the individual tumors
(Fig. 7g). However, there were no significant differences between Co-culture + Ctrl and MDA-MB-231+Ctrl. Immunohistochemistry staining of Ki67, E-cadherin, $\mathrm{N}$-cadherin, Vimentin of the tumors were also conducted. These results showed increased proliferation of Ki67 and enhanced Vimentin and $\mathrm{N}$-cadherin expressions in $\mathrm{Co}-$ culture + TGF- $\beta 1$ compared with the other two groups (Fig. $7 \mathrm{a}-\mathrm{d}$ ). However, the converse was seen with regard to E-cadherin expressions. The HE slides of livers showed no metastasis in all groups, while lung metastasis was observed in Co-culture + TGF- $\beta 1$ and Co-culture + Ctrl. Besides, metastasis areas in co-culture + TGF- $\beta 1$ was more larger than the co-culture $+\mathrm{Ctrl}$ group. No metastasis was seen in the remaining groups (Fig. 7e).

\section{Discussion}

Mesenchymal cell exhibit augmented migratory and procarcinogenic phenotypes. This EMT process allows epithelial cells to lose their cell-cell adhesive qualities and acquire mesenchymal properties. It therefore comes as no surprise that the EMT process is thought to be a critical instigator of the transformation from in situ to invasive carcinoma [18]. Nevertheless, literatures supporting this theory are scarce, with only a few studies investigating the different EMT marker profiles between DCIS and invasive carcinomas. TGF- $\beta 1$ and c-met expressions were raised during the evolution of DCIS to invasive carcinoma, as documented by Logullo et al. [19]. Knudsen et al. [10] has recently demonstrated an upregulation of myoepithelial cell-specific genes and EMT-associated genes in the epithelial cells of invasive carcinoma in comparison to DCIS, suggesting that molecular reprogramming of the epithelial cells are vital in triggering carcinogenesis. Expressions of SMA and $\mathrm{N}$-cadherin, rates of $\beta$-catenin were all markedly raised as well as E-cadherin was reduced in invasive carcinomas when compared to pure DCIS [20]. In line with all these studies, our results also demonstrated that there was a significantly higher rate of E-cadherin loss and higher expressions of $\mathrm{N}$-cadherin, Snail, Twist, Vimentin and Zeb1 in invasive carcinoma in comparison to DCIS.

While EMT has been implicated in tumor invasion partly by reducing cell-cell adhesion, the myoepithelial phenotype has been reported to increase cell motility [21]. Myoepithelial cells surrounding DCIS were found to possess significant epigenetic, genetic and molecular 


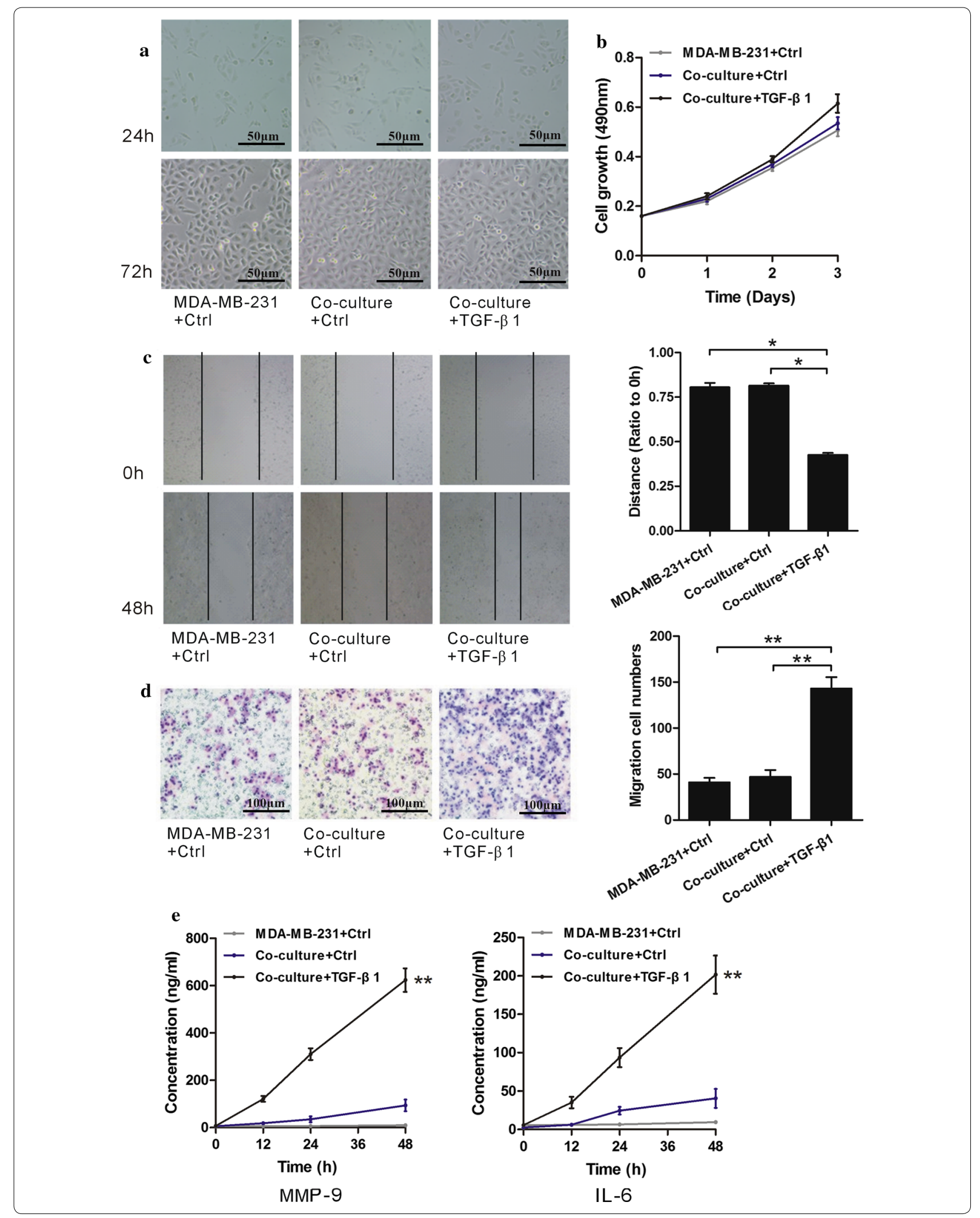




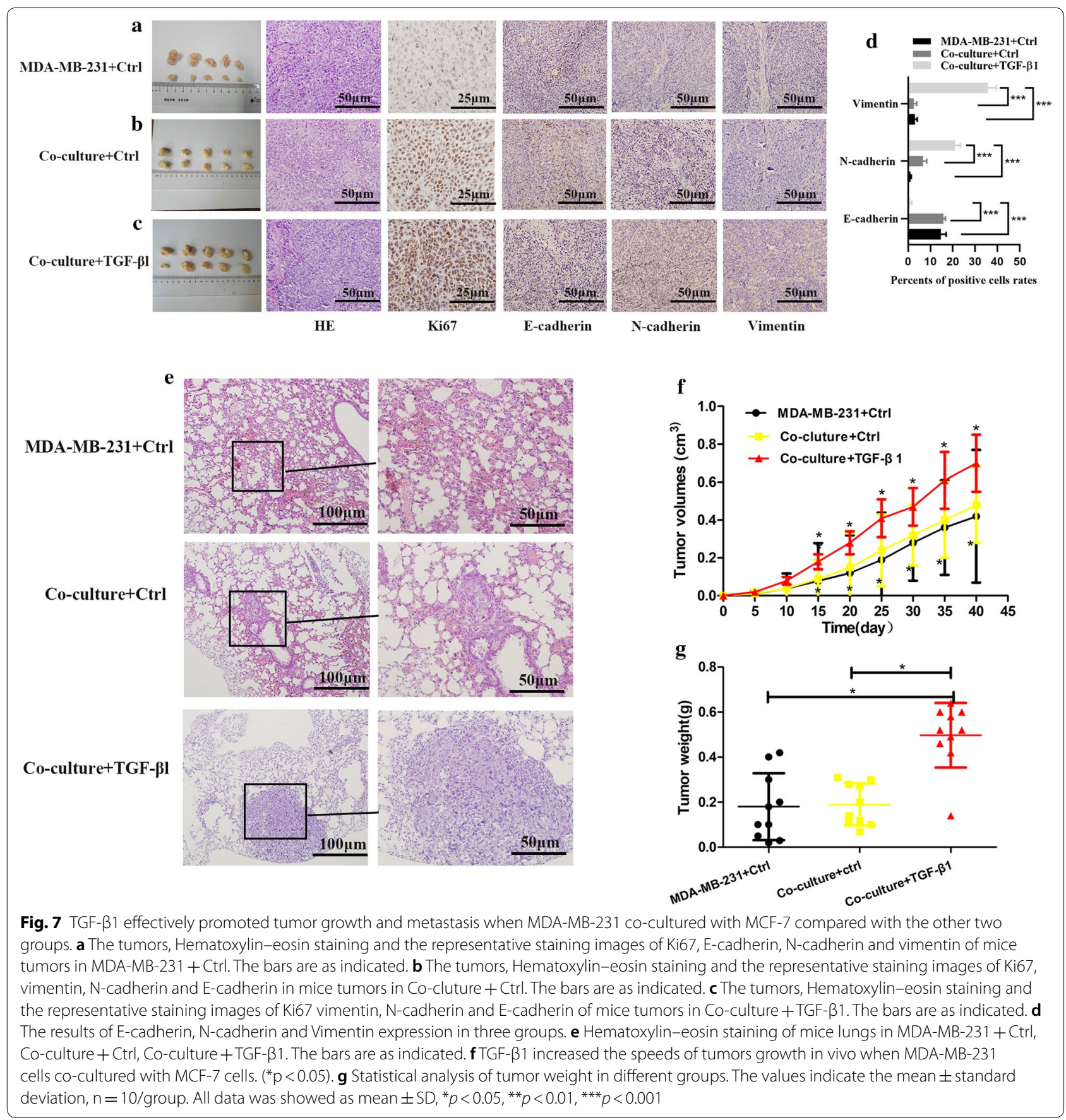

differences in comparison to those in the vicinity of normal breast lobules and ducts [22]. Significant genetic changes include an upregulation of genes for chemokines that augment epithelial cell invasion, migration and proliferation and a downregulation of genes controlling normal myoepithelial cell characteristics [14]. 13.3\% of specimens of DCIS and all of the IDC samples in our study demonstrated no p63 staining, a finding that was also seen in Werling et al. who demonstrated a discontinuous p63 staining pattern in 10\% of DCIS cases [23]. Myoepithelial cells associated with noninvasive proliferations, vascular smooth muscle and normal breast tissue myoepithelial cells all demonstrated high calponin expressions [24]. A study by Hilson et al. found that the myoepithelial cells of DCIS had suppressed calponin expressions [16], a finding that is mirrored in our study. 
Russell et al. reported that the earliest indication of a compromised myoepithelium was the loss of p63, followed by calponin loss at the intermediate stages [25], and SMA changes being the latest marker. These results lay the foundation for further research into the influence of myoepithelial cells on DCIS progression to invasive carcinoma.

TGF- $\beta 1$ stimulation on MCF-7 allowed us to establish an EMT model, which in turn enabled us to demonstrate the EMT effect of MCF-7 on MDA-MB-231 in a co-culture system. TGF- $\beta 1$ indeed regulates EMT though inhibition of MCF-7 cell motility and compromising cellcell adhesion. The EMT is marked by an increase in mesenchymal markers (N-cadherin and Vimentin) and by a loss of epithelial markers (E-cadherin) [26]. These results mirror those of existing literature. TGF- $\beta 1$ also regulated the migration of MCF-7, a feature that did not appear to depend on its general impact on cellular proliferation. To understand the in vitro mechanisms of epithelial cell and myoepithelial cell, a co-culture system allowing the interaction of myoepithelial and epithelial cells (a condition mimicking the tumor microenvironment) was created. This was done by using Transwell chambers, which allowed separation of cells via a porous membrane that allowed free diffusion of soluble factors. Martinez et al. recorded that medium conditioned with malignant epithelial cell was able to induce myoepithelial cell FGF2 secretion that supports carcinogenesis [27], Our results suggest that TGF- $\beta 1$ increases MDA-MB-231 migration, invasion and cytokines secretion when co-cultured with MCF-7. Simultaneous silencing of MMP-9 in breast cancer cells decreased the adhesiveness, invasive, migratory and wound healing characteristics of cells [28]. Allinen et al. found that DCIS-associated myoepithelial cells upregulate the synthesis of enzymes linked to the breakdown of ECM and BM such as MMPs [29]. In agreement with their study, our results suggested that MMP-9 secretion increased when MCF-7 stimulated with TGF- $\beta 1$ and co-cultured with MDA-MB-231. Martinez et al. showed that myoepithelial cells produce IL- 6 in in situ condition [30]. In particular, IL-6 levels peaked after a day period of cell culture. In agreement with their study, our results suggest that IL-6 secretion increases when MCF-7 stimulated with TGF- $\beta 1$ and co-cultured with MDA-MB-231.

Although we have shown that stimulation with TGF- $\beta 1$ bought about EMT in breast cancer, the exact domains and other molecules involved in this interaction remain to be clarified. Additional studies are necessary to further elucidate the effects of MDA-MB-231 on MCF-7 in the co-culture system. We conducted animal studies to further validate the in vivo role of EMT in the progression of in situ to invasive carcinoma. These results demonstrated that the existence of TGF- $\beta 1$ in a MDA-MB-231 co-culture with MCF-7 resulted in EMT promotion, which in turn lead to increased tumor growth and metastatic capability to the lungs. Nevertheless, this coculture system has its potential limitations, given the dynamics and complexity of epithelial cell-myoepithelial cell interactions in the in vivo microenvironment. While soluble factors are able to freely diffuse through the porous membrane of this system, direct cellular interaction is not present. Additionally, our system does not take into account the presence of other genetic mutations that may influence proliferation. These factors may also lead to the differences in cell survival across different coculture groups.

\section{Conclusion}

Taken together, our study demonstrated that there was an increase of mesenchymal marker expression as well as a higher rate of loss of myoepithelial phenotypic markers and loss of E-cadherin in invasive carcinomas when compared to normal healthy breast tissue and DCIS. Most importantly, TGF- $\beta 1$ was critical in regulating the morphology, migration, and EMT markers of MCF-7.TGF- $\beta 1$ regulates migration, invasion, and cytokines secretion of MDA-MB-231 when co-cultured with MCF-7 in vivo subcutaneous tumor formation and metastasis. These findings indicated that EMT phenotypes and cancerassociated myoepithelial cell may be present in the evolution of in situ to invasive breast cancer.

\section{Abbreviations}

DCIS: ductal carcinoma in situ; IDC: invasive ductal carcinoma; TGF- $\beta 1$ : transforming growth factor- $\beta$; EMT: epithelial-mesenchymal transition; ECM: extracellular matrix; BM: basement membrane; FGF2: fibroblast growth factor 2; MMPs: matrix metalloproteinases.

\section{Acknowledgements}

The present study was supported by grants from the National Natural Science Foundation of China (81470119, 81602340 of Shuling Wang) and the Tianjin Municipal Science and the Technology Commission Research Fund (15JCYBJC27800).

\section{Authors' contributions}

YN, LW, CX, XL conceived and designed the experiments. LW, CX and FL performed majority of the experiments. LC and SLW performed the statistical analysis. FL and GMX cultured the cells. QXM, and JL performed some animal experiments. JJ and YY collected the clinical data. LW, CX and YN wrote the manuscript. All authors read and approved the final manuscript.

\section{Funding}

The present study was supported by grants from the National Natural Science Foundation of China $(81470119,81172532,81602340$ of Shuling Wang) and the Tianjin Municipal Science and the Technology Commission Research Fund (15JCYBJC27800).

Availability of data and materials

Please contact the corresponding author for all data requests.

Ethics approval and consent to participate

This study was approved by the Tianjin Medical University Cancer Institute and Hospital, China, and has been performed in accordance with the ethical 
standards laid down in the 1964 Helsinki Declaration and its later amendments. All procedures performed in the studies involving animals were in accordance with the ethical standards of this institution or practice at which the studies were conducted.

\section{Consent for publication}

Informed consent was obtained from all individual participants included in the study.

\section{Conflict of interest}

The authors declare that they have no conflict of interest.

\section{Author details}

${ }^{1}$ Tianjin Medical University Cancer Institute and Hospital, National Clinical Research Center of Cancer, Key Laboratory of Breast Cancer Prevention and Therapy, Tianjin Medical University, Ministry of Education, Key Laboratory of Cancer Prevention and Therapy, West Huanhu Road, Ti Yuan Bei, Hexi District, Tianjin 300060, China. ${ }^{2}$ The Second Department of Breast Cancer, Tianjin Medical University Cancer Institute and Hospital, Tianjin 300060, China. ${ }^{3}$ Department of Breast Cancer Pathology and Research Laboratory, Tianjin Medical University Cancer Institute and Hospital, Tianjin 300060, China. ${ }^{4}$ Department of Breast Oncology, Tianjin Medical University Cancer Institute and Hospital, Tianjin 300060, China. ${ }^{5}$ Department of Oncology, General Hospital of Tianjin Medical University, 154 Anshan Road, Heping District, Tianjin 300052, China.

Received: 15 April 2019 Accepted: 12 December 2019 Published online: 19 December 2019

\section{References}

1. Fu F, Gilmore RC, Jacobs LK. Ductal carcinoma in situ. Surg Clin North Am. 2018;98(4):725-45.

2. Cowell CF, Weigelt B, Sakr RA, Ng CK, Hicks J, King TA, Reis-Filho JS. Progression from ductal carcinoma in situ to invasive breast cancer: revisited. Mol Oncol. 2013;7(5):859-69.

3. Allen MD, Thomas GJ, Clark S, et al. Altered microenvironment promotes progression of preinvasive breast cancer: myoepithelial expression of avß6 integrin in DCIS identifies high-risk patients and predicts recurrence. Clin Cancer Res. 2014;20(2):344-57.

4. Foroni C, Broggini M, Generali D, Damia G. Epithelial-mesenchymal transition and breast cancer: role, molecular mechanisms and clinical impact. Cancer Treat Rev. 2012;38(6):689-97.

5. Lu W, Kang Y. Epithelial-mesenchymal plasticity in cancer progression and metastasis. Dev Cell. 2019;49(3):361-74.

6. Kim YS, Yi BR, Kim NH, Choi KC. Role of the epithelial-mesenchymal transition and its effects on embryonic stem cells. Exp Mol Med. 2014;46:e108

7. Lamouille S, Xu J, Derynck R. Molecular mechanisms of epithelial-mesenchymal transition. Nat Rev Mol Cell Biol. 2014;15(3):178-96.

8. Saitoh M. Epithelial-mesenchymal transition is regulated at post-transcriptional levels by transforming growth factor-beta signaling during tumor progression. Cancer Sci. 2015;106(5):481-8.

9. Suriyamurthy $S$, Baker D, Ten Dijke P, lyengar PV. Epigenetic reprogramming of TGF-beta signaling in breast cancer. Cancers. 2019;11(5):726.

10. Knudsen ES, Ertel A, Davicioni E, Kline J, Schwartz GF, Witkiewicz AK. Progression of ductal carcinoma in situ to invasive breast cancer is associated with gene expression programs of EMT and myoepithelia. Breast Cancer Res Treat. 2012;133(3):1009-24.

11. Lo PK, Zhang Y, Yao Y, Wolfson B, Yu J, Han SY, Duru N, Zhou Q. Tumorassociated myoepithelial cells promote the invasive progression of ductal carcinoma in situ through activation of TGFbeta signaling. J Biol Chem. 2017;292(27):11466-84.

12. Moumen M, Chiche A, Cagnet S, Petit V, Raymond K, Faraldo MM, Deugnier MA, Glukhova MA. The mammary myoepithelial cell. Int J Dev Biol. 2011;55(7-9):763-71.
13. Mao SPH, Park M, Cabrera RM, Christin JR, Karagiannis GS, Oktay MH, Zaiss DMW, Abrams SI, Guo W, Condeelis JS, et al. Loss of amphiregulin reduces myoepithelial cell coverage of mammary ducts and alters breast tumor growth. Breast Cancer Res. 2018;20(1):131.

14. Pandey PR, Saidou J, Watabe K. Role of myoepithelial cells in breast tumor progression. Front Biosci. 2010;15:226-36.

15. Hilson JB, Schnitt SJ, Collins LC. Phenotypic alterations in ductal carcinoma in situ-associated myoepithelial cells: biologic and diagnostic implications. Am J Surg Pathol. 2009;33(2):227-32.

16. Rohilla M, Bal A, Singh G, Joshi K. Phenotypic and functional characterization of ductal carcinoma in situ-associated myoepithelial cells. Clin Breast Cancer. 2015;15(5):335-42.

17. Xu C, Liu F, Xiang G, et al. $\beta$-Catenin nuclear localization positively feeds back on EGF/EGFR-attenuated AJAP1 expression in breast cancer. J Exp Clin Cancer Res. 2019;38(1):238.

18. Sikandar SS, Kuo AH, Kalisky T, Cai S, Zabala M, Hsieh RW, Lobo NA, Scheeren FA, Sim S, Qian D, et al. Role of epithelial to mesenchymal transition associated genes in mammary gland regeneration and breast tumorigenesis. Nat Commun. 2017;8(1):1669.

19. Logullo AF, Nonogaki S, Pasini FS, Osório CA, Soares FA, Brentani MM. Concomitant expression of epithelial-mesenchymal transition biomarkers in breast ductal carcinoma: association with progression. Oncol Rep. 2010;23(2):313-20.

20. Choi Y, Lee HJ, Jang MH, Gwak JM, Lee KS, Kim EJ, Kim HJ, Lee HE, Park SY. Epithelial-mesenchymal transition increases during the progression of in situ to invasive basal-like breast cancer. Hum Pathol. 2013:44(11):2581-9.

21. Guarino M. Epithelial-mesenchymal transition and tumour invasion. Int J Biochem Cell Biol. 2007;39(12):2153-60.

22. Hu M, Yao J, Cai L, Bachman KE, van den Brule F, Velculescu V, Polyak K. Distinct epigenetic changes in the stromal cells of breast cancers. Nat Genet. 2005;37(8):899-905.

23. Werling RW, Hwang $H$, Yaziji H, Gown AM. Immunohistochemical distinction of invasive from noninvasive breast lesions: a comparative study of p63 versus calponin and smooth muscle myosin heavy chain. Am J Surg Pathol. 2003;27(1):82-90.

24. Hsiao YH, Su YA, Tsai HD, Mason JT, Chou MC, Man YG. Increased invasiveness and aggressiveness in breast epithelia with cytoplasmic p63 expression. Int J Biol Sci. 2010;6(5):428-42.

25. Russell TD, Jindal S, Agunbiade S, Gao D, Troxell M, Borges VF, Schedin P. Myoepithelial cell differentiation markers in ductal carcinoma in situ progression. Am J Pathol. 2015;185(11):3076-89.

26. Wendt MK, Smith JA, Schiemann WP. Transforming growth factor-betainduced epithelial-mesenchymal transition facilitates epidermal growth factor-dependent breast cancer progression. Oncogene. 2010;29(49):6485-98.

27. Martinez EF, Demasi AP, Napimoga MH, Arana-Chavez VE, Altemani A, de Araujo NS, de Araujo VC. In vitro influence of the extracellular matrix in myoepithelial cells stimulated by malignant conditioned medium. Oral Oncol. 2012;48(2):102-9.

28. Moirangthem A, Bondhopadhyay B, Mukherjee M, Bandyopadhyay A, Mukherjee N, Konar K, Bhattacharya S, Basu A. Simultaneous knockdown of UPA and MMP9 can reduce breast cancer progression by increasing cell-cell adhesion and modulating EMT genes. Sci Rep. 2016;6:21903.

29. Allinen M, Beroukhim R, Cai L, Brennan C, Lahti-Domenici J, Huang H, Porter D, Hu M, Chin L, Richardson A, et al. Molecular characterization of the tumor microenvironment in breast cancer. Cancer Cell. 2004;6(1):17-32.

30. Martinez EF, Napimoga MH, Montalli VA, de Araujo NS, de Araujo VC. In vitro cytokine expression in in situ-like areas of malignant neoplasia. Arch Oral Biol. 2013;58(5):552-7.

\section{Publisher's Note}

Springer Nature remains neutral with regard to jurisdictional claims in published maps and institutional affiliations. 